CYSTIC FIBROSIS

\title{
Non-tuberculous mycobacteria in end stage cystic fibrosis: implications for lung transplantation
}

\author{
W Chalermskulrat, ${ }^{*}$ N Sood, " I P Neuringer, T M Hecker, L Chang, M P Rivera, \\ L J Paradowski, R M Aris
}

See end of article for authors' affiliations

......................

Correspondence to: $\operatorname{Dr} N$ Sood, Division of Pulmonary Diseases and Critical Care Medicine, Ohio State University, 473 W 12 th Ave, 201 HLRI, Columbus, $\mathrm{OH} 43210$,

USA; Namita.Sood@ osumc.edu

Received 12 July 2005 Accepted 29 January 2006 Published Online First 6 April 2006
Background: WC and NS contributed equally. Non-tuberculous mycobacteria (NTM) frequently colonise patients with end stage cystic fibrosis (CF), but its impact on the course of the disease following lung transplantation is unknown.

Methods: Lung transplant recipients with CF who underwent lung transplantation at our institution between January 1990 and May $2003(n=146)$ and CF patients awaiting lung transplantation in May 2003 $(\mathrm{n}=31)$ were studied retrospectively.

Results: The prevalence rate of NTM isolated from respiratory cultures in patients with end stage CF referred for lung transplantation was $19.7 \%$, compared with a prevalence rate of $13.7 \%$ for NTM isolates in CF lung transplant recipients. The overall prevalence of invasive NTM disease after lung transplantation was low $(3.4 \%)$ and was predicted most strongly by pre-transplant NTM isolation $(p=0.001$, Fisher's exact test, odds ratio (OR) $6.13,95 \% \mathrm{Cl} 3.2$ to 11.4). This association was restricted to Mycobacterium abscessus ( $p=0.005$, Fisher's exact test, OR 7.45, 95\% Cl 2.9 to 16.9). While NTM disease caused significant morbidity in a small number of patients after transplantation, it was successfully treated and did not influence the post-transplant course of the disease.

Conclusion: The isolation of NTM before transplantation in CF patients should not be an exclusion criterion for lung transplantation, but it may alert the clinician to patients at risk of recurrence following transplantation.
$\mathrm{N}$ on-tuberculous mycobacteria (NTM) are ubiquitous environmental organisms and are opportunistic pathogens seen mostly in patients with impaired systemic immunity or in immunocompetent patients with chronic airway disease such as chronic obstructive pulmonary disease or cystic fibrosis (CF). The recognition of NTM as lung pathogens in immunocompetent hosts is often underappreciated as they cause insidious, subtle, and non-specific symptoms. ${ }^{1}$ This is especially true in CF patients with severe bronchiectasis whose airways are chronically infected with co-existing bacteria that inhibit the in vitro growth of NTM..$^{2-4}$ A recent large prospective multicentre study that applied vigilant screening and the use of appropriate NTM culture techniques showed that NTM are commonly isolated from the airway secretions of CF patients with an overall prevalence of $13 \% .^{5}$ Patients with CF develop NTM diseases restricted to the lung, ${ }^{5-7}$ whereas patients with advanced AIDS develop disseminated NTM disease rarely involving the lung..$^{9}$ This suggests complementary roles for host local lung defence and systemic immune defence against NTM disease.

NTM infections have increasingly been recognised after thoracic organ transplantation ${ }^{10-18}$ with a prevalence of 1.4$6.5 \%$. Cystic fibrosis is the leading indication for bilateral lung transplantations, comprising $31.5 \%$ and $44 \%$ of all adult and paediatric bilateral lung transplantations worldwide, respectively. ${ }^{19}$ A cohort of patients with end stage CF serves as a unique homogeneous group of high risk lung transplant candidates in which to study the pre-transplant risk factors for the development of post-transplant NTM infection/ disease. At the time of transplant surgery the severe bronchiectatic airways harbouring NTM are removed while systemic immunosuppressants are introduced. In addition,

*WC and NS contributed equally.
CF patients universally receive bilateral lung transplants which reduces the possibility of seeding NTM from native tissues. ${ }^{20} \mathrm{CF}$ lung transplant recipients undergo the abrupt transition from a population with local risk factors for NTM disease to a population with a combination of risk factors including altered systemic immune status and inherent changes in the thorax after surgery.

We have assessed the impact of pre-transplant NTM infection on the development of post-transplant NTM disease in a retrospective study.

\section{METHODS \\ Patient population}

All CF patients who underwent lung transplantation at our institution (UNC) between January 1990 and May 2003 $(\mathrm{n}=146)$ and those awaiting lung transplantation in May $2003(\mathrm{n}=31)$ were retrospectively studied. Institutional and referral medical records, microbiology, radiology and pathology databases, and lung transplant files were reviewed in accordance with the guidelines of the Institutional Committee on the Protection of the Rights of Human Subjects. The selection criteria, the operative techniques for the donors and recipients, and the medical management following transplantation have been published previously. ${ }^{11} 2122$

\section{Isolation and identification of non-tuberculous mycobacteria (NTM)}

Respiratory specimens from lung transplant candidates, including sputum and bronchoalveolar lavage (BAL) fluids, were obtained at UNC from 1993 to May 2003 during routine

Abbreviations: BMl, body mass index; CF, cystic fibrosis; FVC, forced vital capacity; $\mathrm{FEV}_{1}$, forced expiratory volume in 1 second; NTM, nontuberculous mycobacteria 
transplant evaluation or, before that time, from the referral sites. Respiratory specimens from lung transplant recipients (including sputum, bronchial wash, BAL fluids, lung tissue biopsies, and/or pleural fluids) and donors (bronchial washes immediately after closure) were routinely processed and examined for the presence of NTM. Protocols for specimen processing, culture, and identification of NTM have been described previously. ${ }^{3-5}$ NTM that were difficult to identify were sent to the state reference laboratory for identification. Mycobacterium avium, $M$ intracellulare, and related strains were cumulatively reported as $M$ avium complex.

\section{NTM isolation and disease definitions}

Any positive NTM culture was considered an isolate regardless of AFB smear positivity. A negative isolation was defined as having at least two no-growth culture results. The disease caused by NTM was diagnosed according to the American Thoracic Society guidelines ${ }^{23}$ which include constitutional symptoms (cough, fever, chills, malaise), radiographic changes such as new infiltrates, nodules, bacteriological criteria, positive culture, with or without evidence of granulomatous inflammation in the lung tissue. The CF clinicians made the decisions about NTM treatment and response before transplantation following the best available literature at that time, and the lung transplant physicians (usually in consultation with the CF clinicians) managed the patients after transplantation.

Bronchiolitis obliterans syndrome (BOS) was diagnosed according to the International Society for Heart and Lung Transplantation (ISHLT) criteria. ${ }^{24}$ Pulmonary function testing was performed as previously described. ${ }^{25}$ After NTM disease was diagnosed, empirical broad spectrum antibiotics were initiated based on the speciation according to ATS guidelines. $^{23}$ The drug regimen was clarithromycin or azithromycin, cefoxitin, and amikacin for $M$ abscessus, a quinolone was substituted for the amikacin in case of renal toxicity. A regimen of isoniazid, quinolone, rifabutin and ethambutol was used to treat $M$ kansasii. No significant drug interactions or adverse events were noted.

\section{Statistical analyses}

Non-normally distributed data were presented as medians and ranges or $95 \%$ confidence intervals. Student's $t$ test was performed on normally distributed data to determine if there were any differences in the baseline characteristics of end stage CF patients who did and did not have NTM isolated before transplantation. Non-parametric tests were used for non-normally distributed data. Fisher's exact test was used to analyse the association between isolation of NTM before transplantation and NTM isolation and the development of NTM disease after transplantation.

\section{RESULTS}

\section{Patient population}

One hundred and seventy seven lung transplant candidates with end stage CF constituted the study cohort. Respiratory specimens were cultured for NTM before transplantation in 132 (75\%); for the remaining 45, either the cultures were overgrown with bacteria, they were insufficient for processing, or they were not obtained by the referring institution or UNC. The baseline characteristics of the 132 patients are shown in table 1 . They are representative of the entire population of $177 \mathrm{CF}$ lung transplant candidates and did not differ from the 45 patients who did not have NTM cultures taken before transplantation.

\section{NTM in end stage CF patients}

Twenty nine NTM isolates were cultured from the respiratory specimens of $26 \mathrm{CF}$ lung transplant candidates before transplantation (prevalence rate 19.7\%; fig 1 and table 2 ). The characteristics of these 26 NTM positive patients are compared with those of the 106 NTM negative patients in table 1. There were no differences in age, sex, forced vital capacity (\%FVC), forced expiratory volume in l second $\left(\% \mathrm{FEV}_{1}\right)$, body mass index $(\mathrm{BMI})$, or the coexistence of common bacterial pathogens in the CF airway (Pseudomonas aeruginosa, Burkholderia cepacia complex, or Staphylococcus aureus) between the two groups ( $\mathrm{p}>0.2$ for all).

The most common NTM species isolated was $M$ avium complex $(\mathrm{n}=13,45 \%)$, closely followed by $M$ abscessus ( $\mathrm{n}=12,41 \%$; table 2 ). Other species recovered included $M$ gordonae $(\mathrm{n}=2,7 \%), M$ fortuitum $(\mathrm{n}=1,3 \%)$, and one isolate was unidentifiable $(\mathrm{n}=1,3 \%)$.

Six patients had pre-transplant NTM disease which was restricted to the lungs (patients 1, 2, 3, 8, 9, and 25 in table 2). All except one was due to $M$ abscessus. Two patients were successfully treated, three failed to improve after treatment (patients 1, 3, and 9), and one was unable to tolerate the side effects of treatment (patient 2). The symptoms resolved quickly after treatment, but one of the patients needed long term treatment due to recrudescence of infection when antibiotics were withdrawn

\section{Association between pre-transplant and post- transplant NTM isolation}

None of the lung donors had a past medical history of NTM infection nor had they cultured NTM. Among pre-transplant NTM positive patients who underwent transplantation

Table 1 Characteristics of patients with end stage CF

\begin{tabular}{lllll}
\hline & $\begin{array}{l}\text { End stage CF patients } \\
\text { from whom an NTM } \\
\text { culture was obtained } \\
(\mathbf{N}=132)\end{array}$ & $\begin{array}{l}\text { End stage CF patients } \\
\text { with NTM isolates } \\
(\mathbf{N}=26)\end{array}$ & $\begin{array}{l}\text { End stage CF patients } \\
\text { without NTM isolates } \\
(\mathbf{N}=106)\end{array}$ & p value* \\
\hline Age (years) & $27.3(9.1)$ & $28.5(9.4)$ & $27.1(9.1)$ & 0.48 \\
Sex (female) & $61(46 \%)$ & $12(46 \%)$ & $49(45 \%)$ & 0.92 \\
FVC (l) & $1.86(0.70)$ & $1.98(0.74)$ & $1.83(0.69)$ & 0.36 \\
FVC (\%) & $46.3(12.6)$ & $49.1(14.1)$ & $45.5(12.0)$ & 0.27 \\
FEV 1 (l) & $0.92(0.33)$ & $0.98(0.32)$ & $0.90(0.34)$ & 0.29 \\
FEV $(\%)$ & $26.8(8.8)$ & $28.6(7.7)$ & $26.4(9.0)$ & 0.24 \\
BMI (kg/m $\left.{ }^{2}\right)$ & $18.2(3.0)$ & $18.0(2.5)$ & $18.2(3.1)$ & 0.68 \\
Pseudomonas & $100(76 \%)$ & $19(73 \%)$ & $81(76 \%)$ & 0.73 \\
aeruginosa & $25(19 \%)$ & $6(23 \%)$ & $19(18 \%)$ & 0.58 \\
Burkholderia cepacia & $68(51 \%)$ & $9(42 \%)$ & $59(56 \%)$ & 0.23 \\
Staphylococcus aureus & $68 \%$ & \\
\hline
\end{tabular}

Data are shown as mean (SD) or $\mathrm{n}(\%)$.

NTM, non-tuberculous mycobacteria; FVC, forced vital capacity; FEV $_{1}$, forced expiratory volume in 1 second; BMI, body mass index.

*End stage CF patients with NTM isolates $v$ end stage CF patients without NTM isolates. 


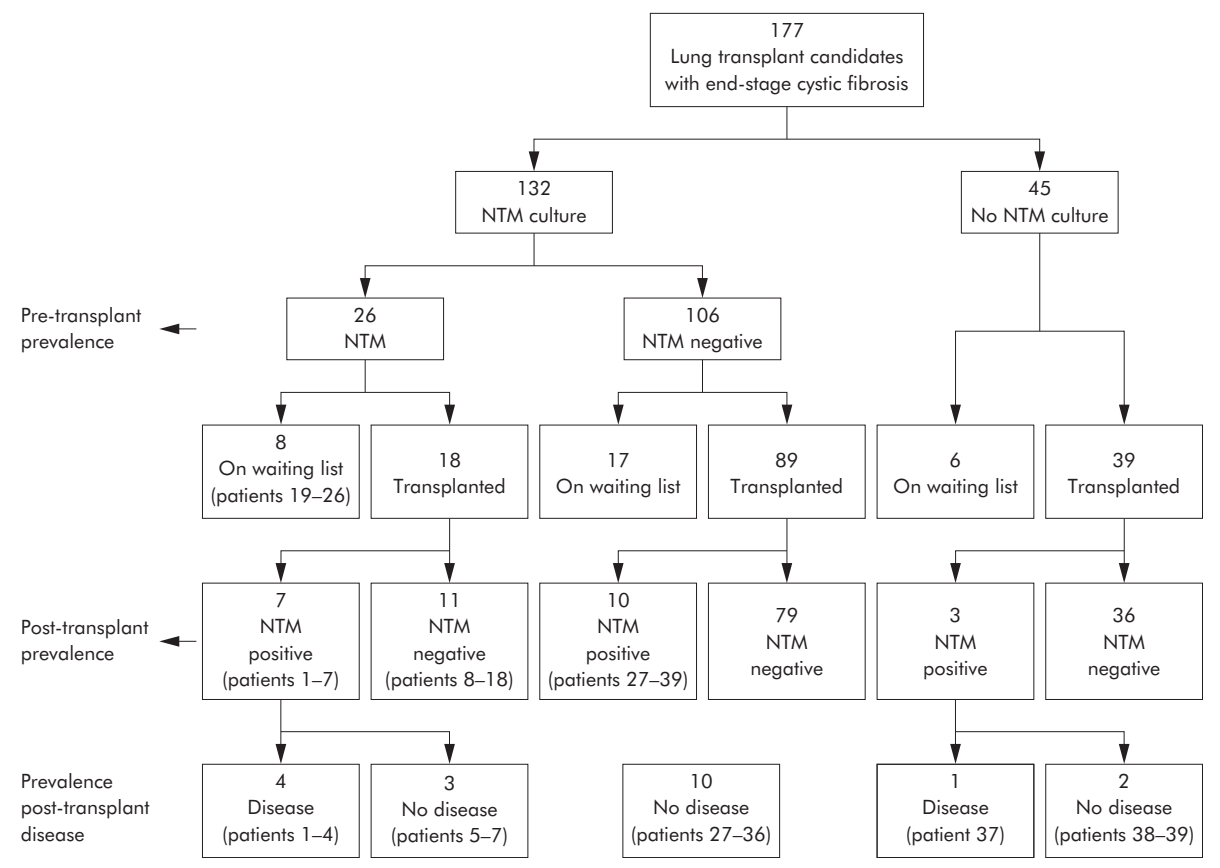

Figure 1 Flow diagram showing the patients with CF included in the study based on the presence of absence of NTM isolation before and after transplantation.

( $\mathrm{n}=18$, fig 1 ; patients $1-18$, table 2 ), seven (39\%) cultured NTM after transplantation. In contrast, NTM was isolated from 10 of $89(11 \%)$ pre-transplant NTM negative patients after transplantation, but none developed NTM disease. There were no differences in any other characteristics including age, sex, \%FVC, \%FEV $\mathrm{FV}_{1}$, BMI, or co-existing colonisation of Pseudomonas spp or Burkholderia cepacia complex between pre-transplant NTM positive and NTM negative transplant recipients $(\mathrm{p}>0.5$ each). Pre-transplant NTM positive CF patients had 5.03 the odds of culturing NTM after lung transplantation compared with pre-transplant NTM negative patients $(p=0.008$, table 3$)$. Of seven pretransplant NTM positive patients from whom NTM were isolated after transplantation (patients 1-7, table 2), the same species was recultured as before transplantation in five ( $M$ abscessus in two (patients 1 and 2), $M$ avium complex in three (patients 3, 5 and 7), table 2).

\section{NTM isolates in CF lung transplant recipients}

One hundred and forty six patients with end stage CF received double or bilateral lung transplants (fig 1). All lung transplant recipients routinely had NTM cultures performed on their respiratory specimens after transplantation at the time of surveillance or diagnostic bronchoscopy. After a mean (SD) post-transplant follow up of 47.1 (42.3) months, 20 transplanted recipients cultured 23 isolates of NTM regardless of pre-transplant NTM status (prevalence 13.7\%; fig 1 and table 2). The NTM were recovered 1-101 months after transplantation (table 2). The most common species isolated from transplanted lungs in CF patients were $M$ avium complex $(\mathrm{n}=9,39 \%), M$ gordonae $(\mathrm{n}=7,30 \%)$, and $M$ abscessus $(\mathrm{n}=3,13 \%)$, followed by $M$ kansasii $(\mathrm{n}=2,9 \%) ; M$ szulgai ( $\mathrm{n}=1,(4 \%)$, and $M$ fortuitum ( $\mathrm{n}=1,4 \%$; table 2 ).

There were no differences in pre-transplant characteristics, including recipient age, $\% \mathrm{FVC}, \% \mathrm{FEV}_{1}, \mathrm{BMI}$, or bacterial colonization in the airway between NTM positive and NTM negative CF lung transplant recipients $(p \geqslant 0.2$ for each characteristic, table 4). The survival of NTM positive CF recipients was not significantly different from that of NTM negative recipients (median 61 (range 11-111), (SD 33.5) months versus 44.7 ( $S D$ 39.5) months; $p=0.06$ ).

\section{NTM disease after lung transplantation}

With a mean post-transplant follow up of 51.8 (35.6) months after the first isolate was identified, five of 20 NTM positive recipients (25\%) developed NTM disease (prevalence of 3.4\% among all CF lung transplant recipients, fig 1). Few NTM species caused disease after lung transplantation ( $M$ abscessus $(\mathrm{n}=3)$ and $M$ szulgai $(\mathrm{n}=1)$, table 2$)$. Only one of $12 M$ avium complex isolates that grew concurrent with $M$ szulgai and one of the two $M$ kansasii caused disease. In contrast, none of the $M$ gordonae $(\mathrm{n}=7)$ or $M$ fortuitum isolates $(\mathrm{n}=1)$ caused disease (table 2). Time of disease onset ranged from 3 to 57 months after transplantation (table 2). The clinical and microbiological features of the patients with post-transplant NTM disease are summarised in table $2(n=5$, patients 1,2 , 3, 7, and 39). Overall, the survival of the five CF lung recipients with post-transplant NTM disease was not different from that of the 141 without post-transplant NTM disease (54.9 (27.4) months $v$ 43.1 (37.2) months; $\mathrm{p}=0.37$ ).

\section{Association between pre-transplant NTM isolation and post-transplant NTM disease}

Among the pre-transplant NTM positive recipients, four (22\%) developed disease (mean (SD) follow up 34.0 (28.2) months after the first isolate was identified). In contrast, none of the pre-transplant NTM negative patients who underwent transplantation $(\mathrm{n}=89)$ developed disease (fig 1). The odds ratio for pre-transplant NTM positive CF patients to develop NTM disease after lung transplantation compared with pre-transplant NTM negative patients was $6.13(\mathrm{p}=0.001$, Fisher's exact test; table 3$)$. Only pretransplant $M$ abscessus positive CF patients were more likely to develop post-transplant disease when compared with pretransplant $M$ abscessus negative patients (odds ratio 7.45; $\mathrm{p}=0.005$, Fisher's exact test; table 3). No significant association was found between pre-transplant infection and post-transplant disease with any other NTM species. 
Table 2 Clinical and microbiological features of patients from whom NTM were isolated before $(n=26)$ and/or after $(n=20)$ transplantation

\begin{tabular}{|c|c|c|c|c|c|c|c|c|c|c|c|c|c|c|}
\hline & \multirow[b]{2}{*}{ Sex } & \multirow[b]{2}{*}{ Age } & \multicolumn{4}{|c|}{ Pre-transplant NTM } & \multicolumn{8}{|c|}{ Post-transplant NTM } \\
\hline & & & Species & Source & $\begin{array}{l}\text { AFB } \\
\text { smear }\end{array}$ & $\begin{array}{l}\text { Time of } \\
\text { isolation } \\
\text { (months } \\
\text { before Tx) }\end{array}$ & Species & Source & $\begin{array}{l}\text { AFB } \\
\text { smear }\end{array}$ & $\begin{array}{l}\text { Time to } \\
\text { isolation } \\
\text { (months } \\
\text { after Tx) }\end{array}$ & $\begin{array}{l}\text { BOS } \\
\text { stage }\end{array}$ & Disease & $\begin{array}{l}\text { Follow up } \\
\text { after NTM } \\
\text { isolation } \\
\text { (months) }\end{array}$ & $\begin{array}{l}\text { Survival } \\
\text { after } \\
\text { transplant } \\
\text { (months) }\end{array}$ \\
\hline 1 & $\mathrm{~F}$ & 22 & M abscessus & Sputum & + & 3,33 & Mabscessus & Wound, blood & + & $4,4,5,6$ & 0 & Yes & 90 & 94 \\
\hline 2 & M & 32 & M abscessus & Sputum & +r+ & 4,11 & M abscessus & Wound swab & +++ & $3,4,4$ & 0 & Yes & 50 & 53 \\
\hline 3 & $\mathrm{~F}$ & 22 & MAC & Sputum & - & 12 & $\begin{array}{l}\text { MAC } \\
\text { M szulgai }\end{array}$ & $\begin{array}{l}\text { Sputum, BAL } \\
\text { Sputum, BAL }\end{array}$ & $\begin{array}{l}++ \\
++\end{array}$ & $\begin{array}{l}38,41 \\
38,41\end{array}$ & $\begin{array}{l}3 \\
3\end{array}$ & Yes & 6 & 44 \\
\hline 4 & M & 29 & Unspecified & Sputum & + & 20 & M kansasii & BAL & ++ & $8,10,10$ & 0 & Yes & 8 & 19 \\
\hline 5 & $\mathrm{~F}$ & 19 & MAC & Sputum & - & 14 & MAC & Sputum, BAL & - & $69,69,69$ & 2 & No & 28 & 97 \\
\hline 6 & $\mathrm{~F}$ & 17 & MAC & Sputum & - & 21 & M gordonae & BAL & - & 9 & 0 & No & 60 & 69 \\
\hline 7 & $M$ & 25 & MAC & Sputum & - & 42 & $\begin{array}{l}\text { MAC } \\
\text { M fortuitum }\end{array}$ & $\begin{array}{l}\text { BAL } \\
\text { BAL }\end{array}$ & $\begin{array}{l}- \\
-\end{array}$ & $\begin{array}{l}18 \\
11,13,18\end{array}$ & $\begin{array}{l}0 \\
0\end{array}$ & $\begin{array}{l}\text { No } \\
\text { No }\end{array}$ & $\begin{array}{l}24 \\
31\end{array}$ & 42 \\
\hline 8 & $\mathrm{~F}$ & 20 & M abscessus & Sputum & H+ & 1 & Negative & - & - & - & - & - & - & 28 \\
\hline 9 & $\mathrm{~F}$ & 26 & M abscessus & Sputum, BAL & H+ & $\begin{array}{l}\text { Persistent*, } \\
29-7\end{array}$ & Negative & - & - & - & - & - & - & 3 \\
\hline 10 & $\mathrm{~F}$ & 31 & M abscessus & Sputum & + & 5,6 & Negative & - & - & - & - & - & - & 23 \\
\hline 11 & M & 19 & M abscessus & Sputum, BAL & + & $\begin{array}{l}22,22,22, \\
24\end{array}$ & Negative & - & - & - & - & - & - & 8 \\
\hline 12 & M & 29 & M abscessus & Sputum & + & $\begin{array}{l}\text { Persistent*, } \\
34-2\end{array}$ & Negative & - & - & - & - & - & - & 21 \\
\hline 13 & $\mathrm{~F}$ & 27 & MAC & Sputum & - & 13 & Negative & - & - & - & - & - & - & 23 \\
\hline 14 & $\mathrm{~F}$ & 36 & MAC & Sputum & + & 26,38 & Negative & - & - & - & - & - & - & 0 \\
\hline 15 & M & 41 & MAC & Sputum, BAL & +r+ & $\begin{array}{l}\text { Persistent*, } \\
41-7\end{array}$ & Negative & - & - & - & - & - & - & 62 \\
\hline 16 & $\mathrm{~F}$ & 18 & $\begin{array}{l}\text { M gordonae } \\
\text { MAC }\end{array}$ & $\begin{array}{l}\text { Sputum } \\
\text { Sputum }\end{array}$ & N/A & $\begin{array}{l}31 \\
9\end{array}$ & Negative & - & - & - & - & - & - & 19 \\
\hline 17 & M & 32 & $\begin{array}{l}\text { M chelonei } \\
\text { abscessus }\end{array}$ & Sputum & N/A & 6 & Negative & - & - & - & - & - & - & 18 \\
\hline 18 & M & 22 & $\begin{array}{l}\text { MAC } \\
\text { M fortuitum }\end{array}$ & $\begin{array}{l}\text { Sputum } \\
\text { Sputum }\end{array}$ & $\begin{array}{l}+ \\
-\end{array}$ & $\begin{array}{l}34,36 \\
41\end{array}$ & Negative & - & - & - & - & - & - & 62 \\
\hline 19 & M & 25 & M abscessus & Sputum & + & On Tx list & - & - & - & - & - & - & - & N/A \\
\hline 20 & $F$ & 14 & M abscessus & Sputum & H+ & On Tx list & - & - & - & - & - & - & - & N/A \\
\hline 21 & M & 48 & M abscessus & Sputum & H+ & On Tx list & - & - & - & - & - & - & - & N/A \\
\hline 22 & M & 35 & MAC & Sputum & - & On Tx list & - & - & - & - & - & - & - & N/A \\
\hline 23 & M & 38 & MAC & Sputum & - & On Tx list & - & - & - & - & - & - & - & N/A \\
\hline 24 & M & 49 & MAC & Sputum & - & On Tx list & - & - & - & - & - & - & - & N/A \\
\hline 25 & $\mathrm{~F}$ & 43 & $\begin{array}{l}\text { M abscessus } \\
\text { MAC }\end{array}$ & $\begin{array}{l}\text { Sputum } \\
\text { Sputum }\end{array}$ & $\begin{array}{l}++ \\
-\end{array}$ & On Tx list & - & - & - & - & - & - & - & N/A \\
\hline 26 & M & 33 & M gordonae & Sputum & - & On Tx list & - & - & - & - & - & - & - & N/A \\
\hline 27 & $\mathrm{~F}$ & 13 & Negative & Sputum & - & - & MAC & BAL & - & 4 & 0 & No & 25 & 29 \\
\hline 28 & $\mathrm{~F}$ & 26 & Negative & Sputum & - & - & MAC & BAL & - & 35 & 3 & No & 4 & 39 \\
\hline 30 & M & 33 & Negative & Sputum & - & - & MAC & BAL & - & 18 & 0 & No & 54 & 72 \\
\hline 29 & M & 21 & Negative & Sputum & - & - & M gordonae & BAL & - & 84 & 2 & No & 5 & 89 \\
\hline 31 & $\mathrm{~F}$ & 24 & Negative & Sputum & - & - & M gordonae & BAL & - & 10 & 0 & No & 17 & 27 \\
\hline 32 & $\mathrm{~F}$ & 29 & Negative & Sputum & - & - & M gordonae & BAL & - & $\begin{array}{l}\text { Persistent*, } \\
8-31\end{array}$ & 0 & No & 51 & 59 \\
\hline 33 & $M$ & 36 & Negative & Sputum & - & - & $M$ gordonae & BAL & - & 2 & 0 & No & 100 & 102 \\
\hline 34 & M & 46 & Negative & Sputum & - & - & M gordonae & BAL & - & 3 & 0 & No & 77 & 80 \\
\hline 35 & M & 29 & Negative & Sputum & - & - & $\begin{array}{l}\text { MAC } \\
\text { M gordonae }\end{array}$ & $\begin{array}{l}\text { BAL } \\
\text { BAL }\end{array}$ & - & $\begin{array}{l}8,14 \\
26\end{array}$ & $\begin{array}{l}0 \\
0\end{array}$ & $\begin{array}{l}\text { No } \\
\text { No }\end{array}$ & $\begin{array}{l}24 \\
6\end{array}$ & 32 \\
\hline 36 & M & 29 & Negative & Sputum & - & - & M kansasii & BAL & + & 1 & 0 & No & 33 & 34 \\
\hline 37 & M & 31 & Unknown & N/A & N/A & N/A & MAC & Sputum & - & 117 & 2 & No & 7 & 124 \\
\hline 38 & $\mathrm{~F}$ & 24 & Unknown & N/A & N/A & N/A & MAC & BAL, wash & - & 101,105 & 3 & No & 30 & 135 \\
\hline 39 & $M$ & 27 & Unknown & N/A & N/A & N/A & $M$ abscessus & Sputum, BAL & - & 57 & 3 & Yes & 13 & 69 \\
\hline
\end{tabular}

\section{Patients who developed NTM disease after lung transplantation}

After NTM disease was diagnosed, empirical antibiotic regimens were initiated based on the speciation according to ATS guidelines ${ }^{23}$ and the clinical details are summarised in table 5. Full details of these patients are available online at http://www.thoraxjnl.com/supplemental.

\section{DISCUSSION}

NTM were isolated from patients with end stage CF who were lung transplant candidates and, to a lesser extent, from CF lung transplant recipients, with prevalence rates of $19.7 \%$ and $13.7 \%$, respectively. While post-transplant NTM disease caused significant morbidity in a small number of patients (prevalence 3.4\%), these patients were successfully treated and the survival of patients with post-transplant NTM (isolation or disease) was not different from those without NTM.

The true prevalence of NTM among end stage CF patients has not previously been determined; in this study we found NTM in approximately one in five patients with end stage CF awaiting lung transplantation. Thus, NTM isolates are more prevalent among end stage CF patients than among a more heterogeneous CF population $(\mathrm{n}=986)$ evaluated in a prospective multicentre study by Olivier et al (prevalence $\sim 13 \%$ ). In both studies the two most common species isolated from CF airways were $M$ avium complex and $M$ abscessus. 
Table 3 Association between pre-transplant NTM isolation and post-transplant NTM isolation and disease

\begin{tabular}{|c|c|c|c|c|}
\hline & $\begin{array}{l}\text { Pre-transplant } \\
\text { NTM positive† } \\
(n=18)\end{array}$ & $\begin{array}{l}\text { Pre-transplant } \\
\text { NTM negative } † \\
\text { ( } \mathrm{n}=89 \text { ) }\end{array}$ & $\begin{array}{l}\text { Pearson } \chi^{2} \\
\text { Odds ratio }\end{array}$ & $p$ value \\
\hline $\begin{array}{l}\text { Post-transplant NTM infection } \\
(n=17)\end{array}$ & 7 (38.9\%) & $10(12.2 \%)$ & 5.03 & $0.003^{*}$ \\
\hline $\begin{array}{l}\text { Post-transplant NTM disease } \\
(n=4)\end{array}$ & $4(22.2 \%)$ & $0(0 \%)$ & 6.13 & $0.001^{*}$ \\
\hline \multirow{2}{*}{$\begin{array}{l}\text { No post-transplant NTM } \\
\text { infection or disease }(n=86)\end{array}$} & $7(38.9)$ & $79(88.8)$ & 0.177 & $0.004^{*}$ \\
\hline & $\begin{array}{l}\text { Pre-transplant } M \\
\text { abscessus positive } † \\
(\mathrm{n}=7)\end{array}$ & $\begin{array}{l}\text { Pre-transplant } M \\
\text { abscessus negative } † \\
\text { (n=100) }\end{array}$ & & \\
\hline $\begin{array}{l}\text { Post-transplant } M \text { abscessus } \\
\text { disease }(n=4)\end{array}$ & $2(28.6 \%)$ & $0(0 \%)$ & 7.45 & $0.005^{*}$ \\
\hline
\end{tabular}

*Fisher's exact test.

†Among those who had NTM cultures pre-transplant.

Following lung transplantation there have been only four previous case reports of NTM species isolated from the airways of CF recipients in the setting of progressive graft dysfunction. Here we report 20 additional CF lung recipients with post-transplant pulmonary NTM isolates and confirm the broad array of clinical settings in which the isolation of NTM can occur. Moreover, the $13.7 \%$ prevalence rate of NTM isolates among our CF patients after transplant is 2-3 times higher than in the general (CF and non-CF) lung transplant population, ${ }^{15}{ }^{16}$ indicating that CF patients are particularly prone to post-transplant NTM infection. We found that the only pre-transplant predictor for post-transplant NTM isolation or disease was pre-transplant NTM isolation, with pretransplant NTM positive patients five times more likely to culture NTM species and six times more likely to develop NTM disease after lung transplantation than pre-transplant NTM negative patients. The similarity of the NTM species recovered before and after transplantation and the lack of their recovery from donor lungs in our study suggests that recurrence of NTM after transplantation was due to colonisation of the native CF airway above the surgical anastomoses (main stem bronchi, trachea or sinuses). The two patients with $M$ abscessus in their explanted (whole) hilar lymph nodes indicates that other reservoirs of NTM exist beyond the respiratory tract in patients with CF, and these may be potential sources of post-transplant infection if intraoperative contamination occurs.

Pulmonary ${ }^{10-13} 151618$ and extrapulmonary ${ }^{15-17}$ diseases caused by NTM have increasingly been recognised following thoracic organ transplantation. Without a consensus, the diagnosis of pulmonary NTM disease and its differentiation from colonisation in immunocompromised lung transplant patients represents a significant challenge. Transplant pulmonologists often treat isolates even if there is uncertainty of invasive disease. In this study, with a mean follow up of 4 years after recovery of the first isolate, only five of $146 \mathrm{CF}$ lung recipients (3.4\%) developed definite NTM disease, all of which involved the thorax. However, four of the patients from whom NTM had been isolated before transplantation developed NTM disease after surgery (three of whom were infected with $M$ abscessus). Only one patient ( $<1 \%$ ) developed disseminated disease. Thus, NTM isolation after transplantation in individuals with CF is common, but rates of invasive disease are low.

Four cases of $M$ abscessus disease have been reported in general lung transplant recipients manifesting as empyema with $(n=1)$ or without $(n=2)^{15}{ }^{18}$ mycobacteraemia or graft dysfunction $(\mathrm{n}=1),{ }^{13}$ resulting in death due to ventilatory failure. ${ }^{18} 26$ We extended these findings by confirming the particular virulence of $M$ abscessus and its ability to recur after transplantation. Pre-transplant $M$ abscessus culture positive CF patients, regardless of AFB smear positivity, were more likely to develop post-transplant disease than pre-transplant $M$ abscessus culture negative patients. Thus, a recurrent isolation of $M$ abscessus from a CF lung transplant recipient should increase the suspicion of the development of disease until proved otherwise. In addition, we observed two distinct presentations by $M$ abscessus after lung transplantation: early infections that caused empyema or mediastinitis, and late infection in the setting of severe lung graft dysfunction (BOS

\begin{tabular}{|c|c|c|c|}
\hline & $\begin{array}{l}\text { Lung transplant recipients } \\
\text { with a post-transplant } \\
\text { NTM isolate } \\
(\mathrm{N}=20)\end{array}$ & $\begin{array}{l}\text { Lung transplant recipients } \\
\text { without a post-transplant } \\
\text { NTM isolate } \\
(\mathrm{N}=126)\end{array}$ & $p$ value \\
\hline Age at transplant (years) & $26.7(7.3)$ & $26.5(8.5)$ & 0.9 \\
\hline Sex (female) & $8(40 \%)$ & $61(48 \%)$ & 0.65 \\
\hline FVC (I) & $1.97(0.70)$ & $1.68(0.64)$ & 0.09 \\
\hline FVC (\%) & $45.7(15.5)$ & $43.2(12.1)$ & 0.52 \\
\hline $\mathrm{FEV}_{1}(\mathrm{l})$ & $0.94(0.3)$ & $0.84(0.3)$ & 0.20 \\
\hline $\mathrm{FEV}_{1}(\%)$ & $25.6(7.5)$ & $25.2(8.7)$ & 0.80 \\
\hline $\mathrm{BMI}\left(\mathrm{kg} / \mathrm{m}^{2}\right)$ & $17.4(2.3)$ & $17.7(2.5)$ & 0.70 \\
\hline Pseudomonas aeruginosa & $12(60 \%)$ & $88(69 \%)$ & 0.54 \\
\hline Burkholderia cepacia & $5(25 \%)$ & $25(20 \%)$ & 0.35 \\
\hline Staphylococcus aureus & $6(30 \%)$ & $53(42 \%)$ & 0.44 \\
\hline
\end{tabular}

Data are shown as mean (SD) or $\mathrm{n}(\%)$.

NTM, non-tuberculous mycobacteria; FVC, forced vital capacity; FEV 1 , forced expiratory volume in 1 second; BMI, body mass index. 
Table 5 Clinical presentation and outcome of patients with post-transplant NTM disease

\begin{tabular}{|c|c|c|c|c|c|c|}
\hline Patient & Diagnosis & NTM & Sensitivities & Treatment & Duration & Outcome \\
\hline 1 & $\begin{array}{l}\text { Sternal wound abscess/ } \\
\text { mediastinitis }\end{array}$ & M abscessus & $\begin{array}{l}\text { Amikacin, azithromycin, } \\
\text { clarithromycin, ciprofloxcin, } \\
\text { cefoxitin }\end{array}$ & $\begin{array}{l}\text { Cefoxitin, } \\
\text { azithromycin, } \\
\text { amikacin }\end{array}$ & 48 months & $\begin{array}{l}\text { Died } 10 \text { years after } \\
\text { transplant from multiple } \\
\text { problems }\end{array}$ \\
\hline 2 & $\begin{array}{l}\text { Sternal wound abscess/ } \\
\text { empyema }\end{array}$ & M abscessus & $\begin{array}{l}\text { Amikacin, azithromycin, } \\
\text { clarithromycin, ciprofloxcin, } \\
\text { cefoxitin }\end{array}$ & $\begin{array}{l}\text { Clarithromycin, } \\
\text { amikacin, cefoxitin }\end{array}$ & 27 months & $\begin{array}{l}\text { Normal graft function at } \\
\text { follow up }\end{array}$ \\
\hline 3 & New pulmonary infiltrates & MAC & Not obtained & $\begin{array}{l}\text { Refused treatment as } \\
\text { had severe BOS }\end{array}$ & & Died \\
\hline 4 & New pulmonary infiltrates & M kansasii & $\begin{array}{l}\text { Amikacin, clarithromycin, } \\
\text { ciprofloxcin, rifabutin, isoniazid, } \\
\text { rifampin, streptomycin, ethambutol }\end{array}$ & $\begin{array}{l}\text { Isoniazid, ciprofloxin, } \\
\text { rifabutin, ethambutol }\end{array}$ & 2 months & $\begin{array}{l}\text { Normal graft function at } \\
\text { follow up }\end{array}$ \\
\hline 39 & $\begin{array}{l}\text { Lung abscess and } \\
\text { mediastinal } \\
\text { lymphadenapathy }\end{array}$ & M abscessus & $\begin{array}{l}\text { Amikacin, azithromycin, } \\
\text { clarithromycin, ofloxacin, } \\
\text { ciprofloxcin, cefoxitin }\end{array}$ & $\begin{array}{l}\text { Clarithromycin, } \\
\text { ofloxacin cefoxitin }\end{array}$ & 11 months & $\begin{array}{l}\text { Died of disseminated } \\
\text { mucomycosis }\end{array}$ \\
\hline
\end{tabular}

stage 3 ) that caused lung abscess. In contrast to a previous report, we did not find that $M$ avium complex was a common cause of post-transplant NTM disease. ${ }^{15}$ In the only case of disease associated with $M$ avium complex in our study, coinfection with $M$ szulgai, a known human pathogen, ${ }^{27}$ may have been exclusively responsible for her deterioration.

These data suggest that the high rate of NTM isolation in CF patients before transplantation results in higher rates of NTM isolation after transplantation but, with the exception of $M$ abscessus, does not increase the rate of NTM disease. While treatment can be challenging, all three cases of $M$ abscessus disease and the one case of $M$ kansasii disease were successfully treated. As a result, the survival of patients with post-transplant NTM isolation or disease was not different from those without. Proactive pre-transplant surveillance for NTM is advocated; the possible benefits from additional intraoperative NTM specific antiseptic practices for these recipients remain unsettled.

This study has potential shortcomings. Retrospective studies can be limited by ascertainment bias, despite our best efforts to review all available paper and electronic records. In addition, we relied on microbiology results over a long time period, so protocols for specimen processing, culture, and identification of NTM may have varied leading us to underestimate the number of cases of isolation and disease. Thirdly, considerable controversy exists over the definitions of isolation, colonisation, infection, and disease which makes it difficult to extrapolate from the non-CF literature and might confound our prevalence statistics. Nonetheless, this is the largest study of NTM in CF patients referred for and following lung transplantation, and our lengthy follow up without the development of disease in cases we defined as "NTM isolation" strengthened our conclusions. Fourthly, as this was a single centre study it was limited by a relatively small number of NTM disease cases and changes in practice over time may have affected the overall results. Lastly, we did not perform molecular genotyping studies to confirm that the cases of $M$ abscessus that occurred early after transplantation were true recurrences rather than new acquisitions. Further study of NTM in patients with CF will be required before treatment recommendations can be made to address NTM positive patients before referral for lung transplantation. In addition, comprehensive studies to assess potential post-transplant risk factors for NTM in both CF and non-CF patients are needed.
In conclusion, NTM are very commonly isolated from the airways of end stage CF lung transplant candidates. CF recipients are at increased risk for NTM isolation and NTM disease after lung transplantation. However, the overall rates of invasive disease are low and the increased risk for posttransplant NTM disease is restricted largely to those with pretransplant $M$ abscessus isolates. Medical treatment effectively controls NTM disease after lung transplantation, resulting in no attributable mortality. The isolation of $M$ abscessus before transplantation was associated with post-transplant disease and morbidity but did not affect survival. We recommend proactive transplant surveillance for patients with $M$ abscessus isolates, and we await an outcomes analysis from other centres to confirm our findings and help us to understand better the full impact of $M$ abscessus on post-transplant survival. Our findings support the view that isolation of $M$ abscessus from the sputum should be considered a relative but not an absolute contraindication for transplantation.

\section{ACKNOWLEDGEMENTS}

The authors thank Steve B Wagoner for his professional graphical assistance and continued support.

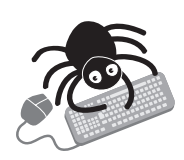

Full details of the five patients who developed NTM disease after lung transplantation are given in the online supplement available at http://www. thoraxinl.com.supplemental.

\footnotetext{
Authors' affiliations W Chalermskulrat, N Sood, I P Neuringer, T M Hecker, L Chang, M P Rivera, L J Paradowski, R M Aris, Lung Transplantation Program and Cystic Fibrosis Research and Treatment Center, Division of Pulmonary Diseases and Critical Care Medicine, University of North Carolina at Chapel Hill, NC, USA

N Sood, Division of Allergy, Pulmonary, Critical Care and Sleep Medicine, The Ohio State University, Columbus, OH, USA

WC is the recipient of an American Lung Association (ALA) Fellowship grant. The authors acknowledge the support of the Cystic Fibrosis Foundation research grants (RMA and WC), ALA research grant (IPN), a National Institute of Health grant HL67178 (IPN), and the Verne S Caviness General Center for Clinical Research (NIH RR00046) (RMA) at UNC-Chapel Hill.

Competing interests: none declared.

This work has been presented in abstract form at the 98th American Thoracic Society International Conference, May 2002, Atlanta, Georgia.
} 


\section{REFERENCES}

1 Falkinham JO. Epidemiology of infection by nontuberculous mycobacteria. Clin Microbiol Rev 1996;9:177-215.

2 Whittier S, Hopfer RL, Knowles MR, et al. Improved recovery of mycobacteria from respiratory secretions of patients with cystic fibrosis. J Clin Microbiol 1993:31:861-4.

3 Whittier S, Olivier K, Gilligan P, et al. Proficiency testing of clinical microbiology laboratories using modified decontamination procedures for detection of nontuberculous mycobacteria in sputum samples from cystic fibrosis patients. The Nontuberculous Mycobacteria in Cystic Fibrosis Study Group. J Clin Microbiol 1997;35:2706-8.

4 Bange FC, Kirschner P, Bottger EC. Recovery of mycobacteria from patients with cystic fibrosis. J Clin Microbiol 1999;37:3761-3.

5 Olivier KN, Weber DJ, Wallace RJ Jr, et al. Nontuberculous mycobacteria: I: Multicenter prevalence study in cystic fibrosis. Am J Respir Crit Care Med 2003;167:828-34

6 Middleton AM, Chadwick MV, Nicholson AG, et al. The role of Mycobacterium avium complex fibronectin attachment protein in adherence to the human respiratory mucosa. Mol Microbiol 2000;38:381-91.

7 Chalermskulrat W, Gilbey JG, Donohue JF. Nontuberculous mycobacteria in women, young and old. Clin Chest Med 2002;23:675-86.

8 Kalayiian RC, Toossi Z, Tomashefski JF Jr, et al. Pulmonary disease due to infection by Mycobacterium avium complex in patients with AIDS. Clin Infect Dis 1995;20:1186-94.

9 Niedt GW, Schinella RA. Acquired immunodeficiency syndrome. Clinicopathologic study of 56 autopsies. Arch Pathol Lab Med 1985; 109:727-34.

10 Chalermskulrat W, Rivera MP. Rapidly developing pulmonary nodules after lung transplantation. Chest 1999;1 16:423S

11 Flume PA, Egan TM, Paradowski $\amalg$, et al. Infectious complications of lung transplantation. Impact of cystic fibrosis. Am J Respir Crit Care Med 1994; 149:1601-7.

12 Kinney JS, Little BJ, Yolken RH, et al. Mycobacterium avium complex in a patient with cystic fibrosis: disease vs. colonization. Pediatr Infect Dis J 1989:8:393-6.

13 Trulock EP, Bolman RM, Genton R. Pulmonary disease caused by Mycobacterium chelonae in a heart-lung transplant recipient with obliterative bronchiolitis. Am Rev Respir Dis 1989; 140:802-5.

14 Baldi S, Rapellino M, Ruffini E, et al. Atypical mycobacteriosis in a lung transplant recipient. Eur Respir J 1997;10:952-4.
15 Malouf MA, Glanville AR. The spectrum of mycobacterial infection after lung transplantation. Am J Respir Crit Care Med 1999;160:1611-6.

16 Kesten S, Chaparro C. Mycobacterial infections in lung transplant recipients. Chest 1999;115:741-5.

17 Torres F, Hodges T, Zamora MR. Mycobacterium marinum infection in a lung transplant recipient. J Heart Lung Transplant 2001;20:486-9.

18 Fairhurst RM, Kubak BM, Shpiner RB, et al. Mycobacterium abscessus empyema in a lung transplant recipient. J Heart Lung Transplant 2002;21:391-4.

19 Hertz MI, Taylor DO, Trulock EP, et al. The registry of the international society for heart and lung transplantation: nineteenth official report-2002. J Heart Lung Transplant 2002;21:950-70.

20 McAdams HP, Erasmus JJ, Palmer SM. Complications (excluding hyperinflation) involving the native lung after single-lung transplantation: incidence, radiologic features, and clinical importance. Radiology 2001;218:233-41.

21 Aris RM, Maia DM, Neuringer IP, et al. Post-transplantation lymphoproliferative disorder in the Epstein-Barr virus-naive lung transplant recipient. Am J Respir Crit Care Med 1996;154:1712-7.

22 Egan TM, Detterbeck FC, Mill MR, et al. Lung transplantation for cystic fibrosis: effective and durable therapy in a high-risk group. Ann Thorac Surg 1998:66:337-46

23 Medical Section of the American Lung Association. Diagnosis and treatment of disease caused by nontuberculous mycobacteria. This official statement of the American Thoracic Society was approved by the Board of Directors. Am J Respir Crit Care Med 1997; 156:S1-25.

24 Estenne M, Maurer JR, Boehler A, et al. Bronchiolitis obliterans syndrome 2001: an update of the diagnostic criteria. J Heart Lung Transplant 2002;21:297-310.

25 Chalermskulrat W, Neuringer IP, Schmitz JL, et al. Human leukocyte antigen mismatches predispose to the severity of bronchiolitis obliterans syndrome after lung transplantation. Chest 2003; 123:1825-31.

26 Sanguinetti $M$, Ardito $F$, Fiscarelli $E$, et al. Fatal pulmonary infection due to multidrug-resistant Mycobacterium abscessus in a patient with cystic fibrosis. J Clin Microbiol 2001;39:816-9

27 Benator DA, Kan V, Gordin FM. Mycobacterium szulgai infection of the lung: case report and review of an unusual pathogen. Am J Med Sci 1997;313:346-51. 